\title{
DARBOUX PROPERTY FOR FUNCTIONS OF SEVERAL VARIABLES
}

BY

\section{J. NEUGEBAUER(1)}

Introduction. Let $E_{n}$ be the $n$-dimensional Euclidean space. A function $f$ : $E_{1} \rightarrow E_{1}$ is said to have the Darboux property if for $a<b$ and $u$ between $f(a)$, $f(b)$ there is $t \in(a, b)$ satisfying $f(t)=u$. There are several important classes of functions which possess the Darboux property: approximately continuous functions and ordinary or approximate derivatives. The purpose of this paper is to extend these results to $E_{n}$ by introducing a Darboux notion for $E_{n}$ with the view of obtaining the following propositions: (1) approximately continuous functions on $E_{n},(2)$ derivatives of certain interval functions, and (3) partial derivatives (approximate or ordinary) of linearly continuous functions possess the Darboux property.

The Darboux property of a function on $E_{1}$ is equivalent with the notion of a connected mapping. The problem then is to define a class of connected sets in $E_{n}$, called Darboux class, relative to which the Darboux property of a function is defined. The Darboux class for $E_{n}$ should contain the connected open subsets of $E_{n}$ and has to be sufficiently large so as to reduce to the Darboux notion for $n=1$, and sufficiently small so as to obtain the propositions (1), (2), and (3). With this in mind the extreme classes, i.e., the class of connected open sets and the class of all connected sets, do not form suitable Darboux classes. For the first class there would be no agreement for $n=1$ [5] and, since an approximately continuous function on $E_{n}(n>1)$ need not be a connected mapping, the second class is too large. In view of the propositions (1), (2), (3) with which this paper is concerned, the Darboux class for $E_{n}$ will depend upon the coordinate system in $E_{n}$.

1. Definition and preliminaries. By an interval $I$ in $E_{n}$ we mean the set of all $x=\left(x_{1}, \cdots, x_{n}\right)$ such that $-\infty<a_{i} \leqq x_{i} \leqq b_{i}<\infty, a_{i}<b_{i}, i=1, \cdots, n$. For $A \subset E_{n},|A|$ denotes the Lebesgue measure of $A, \delta(A)$ stands for the diameter of $A, A^{0}$ is the interior of $A$, and $\bar{A}$ is the closure of $A$.

Let $\{I\}$ be a collection of intervals in $E_{n}$. For $x \in E_{n}$, let $\{I\}_{x}=\{I: I \in\{I\}$ and $x \in I\}$. Let $\phi:\{I\} \rightarrow E_{1}$ be an interval function.

Definition 1. $\phi$ is called continuous at $I_{0} \in\{I\}$ if and only if for every $\varepsilon>0$ there is $\delta>0$ such that for each $I \in\{I\}$ with $\left|I-I_{0}\right|+\left|I_{0}-I\right|<\delta$ there follows $\left|\phi(I)-\phi\left(I_{0}\right)\right|<\varepsilon$.

Received by the editors July 17, 1961 and, in revised form, May 24, 1962.

(1) Supported by NSF Grant No. 18920. 
REMARK. This notion of continuity differs from the one given in [6]. Under some additional hypothesis one is able to select arbitrary small $I_{x} \in\{I\}_{x}$ so that $\phi\left(I_{x}\right)$ is continuous as a function of $x$, a property which is important later.

Definition 2. $\phi$ is termed balanced if and only if for every $I_{0} \in\{I\}$ the following holds. If $I \in\{I\}, I \subset I_{0}$, and $\phi(I) \geqq r_{0} \cdot|I|\left[\phi(I) \leqq r_{0} \cdot|I|\right]$, then there exists $I^{\prime} \in\{I\}$ such that $I^{\prime} \subset I_{0}, \delta\left(I^{\prime}\right) \leqq \delta(I)$, and $\phi\left(I^{\prime}\right) \leqq r_{0} \cdot\left|I^{\prime}\right|\left[\phi\left(I^{\prime}\right) \geqq r_{0} \cdot\left|I^{\prime}\right|\right]$, where $r_{0}=\phi\left(I_{0}\right) /\left|I_{0}\right|$.

EXAMPLES. Let $\{I\}$ be the collection of all intervals in $E_{n}$. Let $\phi(I)=|I|^{2}$ Then $\phi$ is continuous, but not balanced. Let $x_{0} \in E_{n}$, and define $\phi(I)=|I|, x_{0} \in I$, and $\phi(I)=0, x_{0} \notin I$. Then $\phi$ is balanced, but not continuous.

THEOREM 1. If $\{I\}$ is the collection of all intervals of $E_{n}$, and if $\phi:\{I\} \rightarrow E_{1}$ is additive, then $\phi$ is balanced.

Proof. Let $I_{0} \in\{I\}$ and $I \subset I_{0}$ with $\phi(I) \leqq r_{0} \cdot|I|$, where $r_{0}=\phi\left(I_{0}\right) /\left|I_{0}\right|$. Let $\left\{I_{1}, \cdots, I_{K}\right\}$ be a partition of $I_{0}$ with $I_{1}=I$ and $\delta\left(I_{j}\right) \leqq \delta\left(I_{1}\right), j=1, \cdots, K$. If $\phi\left(I_{j}\right)<r_{0} \cdot\left|I_{j}\right|, j=2, \cdots, k$, then $\phi\left(I_{1}\right)+\cdots+\phi\left(I_{K}\right)<r_{0}\left|I_{0}\right|=\phi\left(I_{0}\right)$, contradicting the additivity of $\phi$.

Corollary. If $f: E_{1} \rightarrow E_{1}$ and $\phi([a, b])=f(b)-f(a)$, then $\phi$ is balanced.

Let $\{I\}$ be a collection of intervals of $E_{n}$ and let $x$ be a point in $E_{n}$ for which there is a sequence $\left\{I_{j}\right\} \subset\{I\}_{x}$ with $\delta\left(I_{j}\right) \rightarrow 0$.

Definition 3. An interval function $\phi:\{I\} \rightarrow E_{1}$ has a derivative $\phi^{\prime}(x)$ at $x$ if and only if for $I \in\{I\}_{x}$ and $\delta(I) \rightarrow 0$,

$$
\lim \frac{\phi(I)}{|I|}=\phi^{\prime}(x) .
$$

Let us recall that we wish to study the connected sets that are carried by $\phi^{\prime}$ into connected sets. Some further restrictions on $\phi$ are necessary as the following examples show. Let $\{I\}$ be the collection of all compact intervals of $E_{n}$. Let $A$ be any nondegenerate connected subset of $E_{n}$ and let $x_{0} \in A$. Define $\phi(I)=|I|, x_{0} \in I$, and $\phi(I)=0, x_{0} \notin I$. Then $\phi^{\prime}(x)=0, x \neq x_{0}$, and $\phi^{\prime}\left(x_{0}\right)=1$. Hence $\phi^{\prime}(A)$ is not connected. Note that $\phi$ is balanced, but not continuous. If we let for $I \in\{I\}, d(I)=\operatorname{dist}\left(I, x_{0}\right)$, then the interval function

$$
\phi(I)=|I|^{1+d(I)}
$$

is continuous with $\phi^{\prime}(x)=0, x \neq x_{0}$, and $\phi^{\prime}\left(x_{0}\right)=1$. Again $\phi^{\prime}(A)$ is not connected. We note that $\phi$ is not balanced.

In the sequel, the collection of intervals $\{I\}$ on which $\phi$ is defined has to be sufficiently large. We introduce the following definition.

Definition 4. Let $A \subset E_{n}$. A collection of intervals $\{I\}$ of $E_{n}$ is said to cover $A$ strongly if and only if (1) for each $x \in A$ there is a sequence $\left\{I_{j}\right\} \subset\{I\}_{x}$ such that $\delta\left(I_{j}\right) \rightarrow 0,(2)\{I\}$ contains every interval $I$ with $I^{0} \subset A$. 
2. Darboux sets. Let $Q$ be the generic notation for a set in $E_{n}$ such that $\bar{Q}$ is an interval and $(\bar{Q})^{0} \subset Q \subset \bar{Q}$.

Definition 1. $A$ set $D \subset E_{n}$ will be called a Darboux set if and only if

(1) for every $x \in D$ there is an interval $I$ such that $x \in I$ and $I^{0} \subset D$,

(2) any two points $x, y \in D$ are chained in $D$, i.e., there are $Q_{1}, \cdots Q_{j}$ with $Q_{i} \subset D, i=1, \cdots, j, x \in Q_{1}, y \in Q_{j}$, and $Q_{i} \cap Q_{i+1} \neq \emptyset, i=1, \cdots, j-1$.

It follows that every Darboux set is connected and that every connected open set is a Darboux set. The following example may help to clarify (2). Let $A=\{(x, y)$ : $(x, y) \in E_{2}$ and $y>|x|$ or $\left.y \leqq 0\right\}$. Then $A$ is a connected set satisfying (1), but $(0,0)$ and $(0,1)$ are not chained in $A$.

Definition 2. A function $f: A \rightarrow E_{m}, A \subset E_{n}$, is said to possess the Darboux property on $A$ if and only if $f(D)$ is connected for every Darboux set $D \subset A$.

For the proof of Theorem 2 it is helpful to have the following terminology.

Let $x_{1}, x_{2} \in E_{n}, x_{1} \neq x_{2}$, and let $I_{j}$ be an interval containing $x_{j}, j=1,2$. We term $I_{1}, I_{2}$ equivalent if and only if there is a translation $\tau: E_{n} \rightarrow E_{n}$ with $\tau\left(I_{1}\right)=I_{2}$ and $\tau\left(x_{1}\right)=x_{2}$.

THEOREM 2. Let $A \subset E_{n}$ and let $\{I\}$ cover $A$ strongly. Let $\phi:\{I\} \rightarrow E_{1}$ be continuous, balanced, and differentiable in $A$. Then the derivative $\phi^{\prime}$ has the Darboux property on $A\left({ }^{2}\right)$.

Proof. Let $\bar{Q}$ be an interval, and let $(\bar{Q})^{0} \subset Q \subset \bar{Q}, Q \subset A$. It suffices to show that $\phi^{\prime}(Q)$ is connected. If we deny this, there is a point $x_{0} \in E_{1}$ such that $\phi^{\prime}(Q)$ is the union of two nonempty sets $A^{\prime}, B^{\prime}$ with $A^{\prime} \subset\left\{x: x<x_{0}\right\}, B^{\prime} \subset\left\{x: x>x_{0}\right\}$. Let $A=\left\{p: p \in Q\right.$ and $\left.\phi^{\prime}(p) \in A^{\prime}\right\}, B=\left\{p: p \in Q\right.$ and $\left.\phi^{\prime}(p) \in B^{\prime}\right\}$. Since $Q=A \cup B$, we may assume that $Q^{0} \cap A \neq \varnothing$. Let $a \in Q^{0} \cap A, b \in B$, and join $a, b$ by a segment $\alpha$.

Since $\phi^{\prime}(a)<x_{0}, \phi^{\prime}(b)>x_{0}$, there is an interval $I_{b} \in\{I\}_{b}$ such that $\delta\left(I_{b}\right)<1, I_{b} \subset \bar{Q}, \phi\left(I_{b}\right)>x_{0} \cdot\left|I_{b}\right|$, and if for $p \in \alpha-b, I_{p}$ is the interval in $\{I\}_{p}$ equivalent with $I_{b}$, then $I_{p} \subset Q^{0}$ and $\phi\left(I_{a}\right)<x_{0} \cdot\left|I_{a}\right|$. The function $\phi\left(I_{p}\right)$ is continuous in $p$ on $\alpha$. Hence there is a point $p_{1} \in \alpha$ such that $\phi\left(I_{1}\right)=x_{0} \cdot\left|I_{1}\right|$, where $I_{1}=I_{p_{1}}$. We note that $I_{1} \subset Q^{0}$ and $\delta\left(I_{1}\right)<1$.

Let $a_{1} \in I_{1}^{0}$. We may assume that $a_{1} \varepsilon A$. There is $\frac{1}{2}>\varepsilon>0$ such that if $I \in\{I\}_{a_{1}}$ and $\delta(I)<\varepsilon$, then $I \subset I_{1}$ and $\phi(I)<x_{0} \cdot|I|$. Let then $I \in\{I\}_{a_{1}}$ with $\delta(I)<\varepsilon$. Since $\phi$ is balanced, there is an interval $I^{\prime} \subset I_{1}$ such that $\delta\left(I^{\prime}\right)<\varepsilon$ and $\phi\left(I^{\prime}\right) \geqq x_{0}$. $\left|I^{\prime}\right|$. Let $p^{\prime} \in I^{\prime}$ and join $p^{\prime}, a_{1}$ by a line segment $\alpha_{1}$. If we denote by $I_{p}, p \in \alpha_{1}$, the interval in $\{I\}_{\rho}$ equivalent with $I^{\prime}$, then $I_{p} \subset I_{1}$ and $\phi\left(I_{a_{1}}\right)<x_{0} \cdot\left|I_{a_{1}}\right|$. By the continuity of $\phi$, there is $p_{2} \in \alpha_{1}$ such that $\phi\left(I_{2}\right)=x_{0} \cdot\left|I_{2}\right|$, where $I_{2}=I_{p_{2}}$. We note that $I_{2} \subset I_{1}$ and $\delta\left(I_{2}\right)<\frac{1}{2}$.

Continuing in this manner, we obtain a sequence of intervals $\left\{I_{j}\right\} \subset\{I\}$ such that $Q^{0} \supset I_{1} \supset \cdots \supset I_{j} \supset \cdots, \delta\left(I_{j}\right) \rightarrow 0$, and $\phi\left(I_{j}\right)=x_{0} \cdot\left|I_{j}\right|$. Let $p_{0} \in \cap_{i} I_{j}$. Then $p_{0} \in Q$ and $\phi^{\prime}\left(p_{0}\right)=x_{0}$, a contradiction.

(2) See also [9]. 
THEOREM 3. Let $C$ be a nondegenerate connected subset of $E_{n}$ which is not a Darboux set. Then there exists a collection $\{I\}$ of intervals covering $C$ strongly and a continuous, balanced, and differentiable interval function $\phi:\{I\} \rightarrow E_{1}$ such that its derivative $\phi^{\prime}$ carries $C$ into a disconnected set.

Proof. There are two cases to be considered.

Case 1. There is a point $p_{0} \in C$ such that for every interval $I$ with $p_{0} \in I, I^{0}-C \neq \varnothing$. Let $\{I\}$ be the collection of all intervals $I$ with $p_{0} \notin I$ and the collection of all intervals $I$ with $p_{0} \in I^{0}$. Then $\{I\}$ covers $C$ strongly. Define for $I \in\{I\}, \phi(I)=|I|$, $p_{0} \in I^{0}$, and $\phi(I)=0, p_{0} \notin I$. Then $\phi$ is continuous, balanced, and $\phi^{\prime}(p)=0, p \neq p_{0}$, and $\phi^{\prime}\left(p_{0}\right)=1$. Hence $\phi^{\prime}(C)$ is not connected.

Case 2. For every point $p \in C$ there is an interval $I_{p}$ such that $p \in I_{p}$ and $I_{p}^{0} \subset C$ Let $p_{0} \in C$, and let

$$
\begin{aligned}
& A=\left\{p: p \in C \text { and } p, p_{0} \text { are chained in } C\right\}, \\
& B=\left\{p: p \in C \text { and } p, p_{0} \text { are not chained in } C\right\} .
\end{aligned}
$$

Since $C$ is not Darboux, there follows that $C=A \cup B$ and $A \neq \varnothing, B \neq \varnothing$. We note that for each $p \in C$ the associated interval $I_{p}$ has the property that either $I_{p}^{0} \subset A$ or else $I_{p}^{0} \subset B$.

Let $\{I\}$ be the collection of all intervals $I$ with $I^{0} \subset C$. Then $\{I\}$ covers $C$ strongly. Define $\phi(I)=|I|$, if $I^{0} \subset A$, and $\phi(I)=0$, if $I^{0} \subset B$. Then $\phi^{\prime}(p)=1$, $p \in A$, and $\phi^{\prime}(p)=0, p \in B$, and therefore $\phi^{\prime}(C)$ is not connected. It is easy to see that $\phi$ is continuous and balanced.

Combining Theorems 2 and 3 we obtain the following characterization of Darboux sets.

THEOREM 4. A nondegenerate connected set $D \subset E_{n}$ is a Darboux set if and only if for every collection of intervals $\{I\}$ covering $D$ strongly and for every continuous, balanced, and differentiable $\phi:\{I\} \rightarrow E_{1}$, the derivative $\phi^{\prime}$ carries $D$ into a connected set.

3. Mean value property. Let $\{I\}$ be the collection of all intervals in $E_{n}$ and let $\phi:\{I\} \rightarrow E_{1}$ be a differentiable interval function with derivative $\phi^{\prime}$.

Definition. $\phi$ is said to have the mean value property (simply MV-property) if and only if every $I \in\{I\}$ there is a point $p \in I^{0}$ such that

$$
\frac{\phi(I)}{|I|}=\phi^{\prime}(p) \text {. }
$$

The interval function $\phi(I)=|I|^{2}$ shows that continuity and differentiability are not enough for the MV-property. We note that $\phi$ is not balanced. The interval function defined by $\left(p_{0} \in E_{n}\right) \phi(I)=(1-|I|) \cdot|I|, p_{0} \in I$, and $\phi(I)=0, p_{0} \notin I$, is balanced, and $\phi^{\prime}(p)=0, p \neq p_{0}$, and $\phi^{\prime}\left(p_{0}\right)=1$. Thus $\phi$ does not possess the MV-property. It is clear that $\phi$ is not continuous. We have, however, the following theorem. 
THEOREM 5. Let $\{I\}$ be the collection of all intervals in $E_{n}$, and let $\phi:\{I\} \rightarrow E_{1}$ be continuous, balanced, and differentiable in $E_{n}$. Then $\phi$ has the $M V$-property $\left({ }^{2}\right)$.

Proof. The proof is similar to the proof of Theorem 2. Let $I_{0} \in\{I\}$ and let $\phi\left(I_{0}\right) /\left|I_{0}\right|=x_{0}$. Let $p_{0} \in I_{0}^{0}$, and assume that $\phi^{\prime}\left(p_{0}\right)<x_{0}$. As in the proof of Theorem 2, there is an interval $I_{1} \subset I_{0}^{0}$ such that $\delta\left(I_{1}\right)<1$ and $\phi\left(I_{1}\right)=x_{0} \cdot\left|I_{1}\right|$. Applying the same argument to $I_{1}$ and continuing in this way, we obtain a sequence $I_{0}^{0} \supset I_{1} \supset I_{2} \supset \cdots, \delta\left(I_{j}\right) \rightarrow 0$, and $\phi\left(I_{j}\right)=x_{0} \cdot\left|I_{j}\right|$. For $p \in \cap I_{j}$, we have $p \in I_{0}^{0}$ and $\phi^{\prime}(p)=x_{0}$.

THEOREM 6. Let $\{I\}$ be the collection of all compact intervals of $E_{n}$. Let $\phi:\{I\}$ $\rightarrow E_{1}$ be continuous and differentiable. If $\phi$ possesses the $M V$-property, then its derivative $\phi^{\prime}$ has the Darboux property.

Proof. Let $\bar{Q}$ be an interval in $E_{n}$, and let $(\bar{Q})^{0} \subset Q \subset \bar{Q}$. It suffices to show that $\phi^{\prime}(Q)$ is connected. If this is not the case, we may assume that $Q=A \cup B$, $A \neq \varnothing, B \neq \varnothing$, and $\phi^{\prime}(A) \subset\left\{x: x<x_{0}\right\}, \phi^{\prime}(B) \subset\left\{x: x>x_{0}\right\}$ (see proof of Theorem 2). As in the proof of Theorem 2, there is an interval $I \subset Q$ such that $\phi(I)=x_{0} \cdot|I|$. By the MV-property, there is $p_{0} \in I^{0}$ such that $\phi^{\prime}\left(p_{0}\right)=x_{0}$, a contradiction.

It is known that for functions of a real variable, the MV-property and Darboux property are equivalent (see e.g.[3]). The example $\phi(I)=|I|^{2}$ shows that this is false for interval functions. The next theorem contains some more information of this sort.

THEOREM 7. For continuous, differentiable interval functions $\phi$ with derivative $\phi^{\prime}$ the following holds: balanced $\rightarrow M V$-property $\rightarrow$ Darboux property of $\phi^{\prime}$. None of the implications can be reversed.

Proof. In view of the preceding theorems and remark we need only show that MV-property does not imply "balanced." Let $I_{0}=\left[a_{0}, b_{0}\right]$ be an interval in $E_{1}$. Let $f:\{x: x \geqq 0\} \rightarrow\{x: x \geqq 0\}$ be continuous and have the property that $f(0)=0,0<f(x)<x / 2$ for $x \in\left(0,\left|I_{0}\right|\right)$, and $f(x)=0$ for $x \geqq\left|I_{0}\right|$. For $I$ a compact interval in $E_{1}$, let $d(I)$ be the midpoint of $I$. Define

$$
\phi(I)=\left[\left|d\left(I_{0}\right)-d(I)\right|+f(|I|)\right] \cdot|I| .
$$

It is clear that $\phi$ is continuous and that $\phi^{\prime}(x)=\left|d\left(I_{0}\right)-x\right|$ for $x \in E_{1}$. We will verify that $\phi$ has the MV-property. Let $I$ be an interval. Since $0 \leqq f(|I|)<|I| / 2$, there is a point $x \in I^{0}$ such that

$$
\left|d\left(I_{0}\right)-x\right|=\left|d\left(I_{0}\right)-d(I)\right|+f(|I|) .
$$

Hence

$$
\frac{\phi(I)}{|I|}=\phi^{\prime}(x)
$$


We show next that $\phi$ is not balanced. We have $\phi\left(I_{0}\right)=0$, and, since $0<f(|I|)$ for $|I|<\left|I_{0}\right|$, for every proper subinterval $I$ for $I_{0}$ we have $\phi(I)>0$. This completes the proof.

4. Applications. In this section we will consider some classes of functions which possess the Darboux property.

A function $f: E_{n} \rightarrow E_{1}$ is called approximately continuous in $E_{n}$ if and only if for each $p \in E_{n}$ there is a measureable set $E$ such that $p$ is a point of density one of $E$ and $f \mid E$ is continuous at $p$ (see [6]).

REMARK. We do not make a distinction between ordinary and strong metric density since the theorem below is true for either notion.

THEOREM 8. Iff: $E_{n} \rightarrow E_{1}$ is approximately continuous, then f has the Darboux property.

Proof. We first assume that $f$ is bounded. Then $f$ is summable on every compact interval $I \subset E_{n}$. Let $\phi(I)=\int_{I} f$. It is clear that $\phi$ is continuous, and since $\phi$ is additive, we have by Theorem 1 that $\phi$ is balanced. By [6, p. 132], $\phi^{\prime}(x)=f(x)$, $x \in E_{n}$. By Theorem 2, $\phi^{\prime}=f$ has the Darboux property.

If $f$ is unbounded, let $h: E_{1} \rightarrow(0,1)$ be a homeomorphism. Then $g(x)=h[f(x)]$ is approximately continuous and bounded. Hence $g$ and therefore $f$ has the Darboux property.

REMARK. For further details concerning the class of connected sets that remain connected under approximately continuous transformations the reader should consult $[4 ; 5]$.

THEOREM 9. Let $f: E_{n} \rightarrow E_{1}$. If for each $\left(x_{1}^{0}, \cdots, x_{n}^{0}\right) \in E_{n}$, the functions $f\left(x_{1}^{0}, \cdots, x_{i-1}^{0}, x_{i}, x_{i+1}^{0}, \cdots, x_{n}^{0}\right), i=1, \cdots, n$, possess the Darboux property, then $f$ possesses the Darboux property.

Proof. For notational reasons we will restrict ourselves to $n=2$. It suffices to show that for $\bar{Q}$ an interval and $(\bar{Q})^{0} \subset Q \subset \bar{Q}, f(Q)$ is connected. By hypothesis, every horizontal and vertical line segment in $Q$ is carried by $f$ into a connected set.

Case 1. Every point in $Q$ lies on a horizontal or vertical line segment which is contained in $Q$. In this case it follows from the above observation that $f(Q)$ is connected.

Case 2. There is a point $p_{0}=\left(x_{0}, y_{0}\right)$ in $Q$ such that $p_{0}$ does not lie on a horizontal or vertical line segment which is contained in $Q$. Then it follows that $p_{0}$ is a vertex of $Q$. Let $H$ be a compact horizontal line segment such that $p_{0} \in H, Q^{0} \cup H \subset \bar{Q}$. Since $f(H)$ is connected, there is a sequence $p_{n} \in H-p_{0}$ such that $f\left(p_{n}\right) \rightarrow f\left(p_{0}\right)$. For each $n$, there is a vertical line segment $V_{n}$ such that $p_{n} \in V_{n}$ and $V_{n} \subset \bar{Q}$. Since $f\left(V_{n}\right)$ is connected, there is a point $s_{n} \in V_{n} \cap Q^{0}$ such that $\left|f\left(s_{n}\right)-f\left(p_{n}\right)\right|<1 / n$. Then $f\left(s_{n}\right) \rightarrow f\left(p_{0}\right)$, and therefore $f\left(Q^{0} \cup p_{0}\right)$ is connected This completes the proof. 
REMARK. It is easy to give examples showing that the converse of Theorem 8 is not true.

In the sequel we need the notion of an approximate derivative introduced by A. Denjoy [1]. The properties of approximate derivatives that will be used below can be found in $[7 ; 8]$ with simpler proofs in [3]. In particular, if $f: E_{1} \rightarrow E_{1}$ is an approximate derivative, then $f$ has the MV-property, the Darboux property, and is a function of Baire type at most one.

We need the following known lemma, whose proof is short and will be given for completeness.

Lemma. Let $I=[a, b]$ be an interval in $E_{1}$, and let $A_{1}, A_{2}$ be two nonempty measurable sets such that $I=A_{1} \cup A_{2}$. Then $A_{i}$ contains a point of positive upper density of $A_{j}$ for some $i \neq j, i, j=1,2$.

Proof. Suppose this is not the case. Then $x \in A_{1}$ implies that $x$ is a point of density one of $A_{i}, i=1,2$. Then the characteristic function $c_{1}$ of $A_{1}$ is approximately continuous. By Theorem $8, c_{1}(I)$ should be connected, a contradiction since $c_{1}\left(A_{1}\right)=1, c_{1}\left(A_{2}\right)=0$.

Definition. A function $f: E_{2} \rightarrow E_{1}$ will be called linearly approximately continuous if and only if for each $\left(x_{0}, y_{0}\right) \in E_{2}$, the functions $f\left(x_{0}, y\right), f\left(x, y_{0}\right)$ are approximately continuous as a function of $y, x$, respectively.

THEOREM 10. Let $f: E_{2} \rightarrow E_{1}$ be linearly approximately continuous, and assume that the approximate partial derivative with respect to $x,\left(f_{x}\right)_{a p}$, exists everywhere in $E_{2}$. Then $\left(f_{x}\right)_{a p}: E_{2} \rightarrow E_{1}$ has the Darboux property.

Proof. Let $h=\left(f_{x}\right)_{a p}$. By $[6$, p. 299] $h$ is measurable. Let $I=[a, b] \times[c, d]$. We will first prove that $h(I)$ is connected. If we deny this, there is a point $x_{0} \in E_{1}$ and there are two sets $A \neq \emptyset, B \neq \varnothing$, such that $I=A \cup B$ and $h(A) \subset\left\{x: x<x_{0}\right\}$, $h(B) \subset\left\{x: x>x_{0}\right\}$. For each $y \in[c, d]$, let $H_{y}=\{(x, y): x \in[a, b]\}$. Since $h\left(H_{y}\right)$ is connected, $H_{y} \subset A$ or else $H_{v} \subset B$. Thus there are two sets $A_{1}, A_{2}$ such that $[c, d]=A_{1} \cup A_{2}$, and $A=[a, b] \times A_{1}, B=[a, b] \times A_{2}$. Since $h$ is measurable, $A, B$ are measurable, and, therefore, $A_{1}, A_{2}$ are measurable subsets of $[c, d]$. By the lemma we may assume that there is $y_{0} \in A_{1}$ which is also a point of positive upper density of $A_{2}$.

Since $f(a, y), f(b, y)$ are approximately continuous, and since $y_{0}$ is a point of positive upper density of $A_{2}$, there is a sequence $\left\{y_{n}\right\}$ in $A_{2}$ such that $y_{n} \rightarrow y_{0}$ and $f\left(a, y_{n}\right) \rightarrow f\left(a, y_{0}\right), f\left(b, y_{n}\right) \rightarrow f\left(b, y_{0}\right)$. For each $n, n=0,1,2, \cdots$, there is a point $z_{n} \in(a, b)$ such that

$$
f\left(b, y_{n}\right)-f\left(a, y_{n}\right)=h\left(z_{n}, y_{n}\right)(b-a) .
$$

Therefore, $h\left(x_{n}, y_{n}\right) \rightarrow h\left(z_{0}, y_{0}\right)$. Since $\left(z_{n}, y_{n}\right) \in B, h\left(z_{n}, y_{n}\right)$ is in $h(B)$. Therefore $h\left(z_{0}, y_{0}\right)>x_{0}$, contradicting $\left(z_{0}, y_{0}\right) \in A$. Thus $h(I)$ is connected. 
To complete the proof, it suffices to show that for every set $Q$, satisfying $(\bar{Q})^{0} \subset Q \subset \bar{Q}, \bar{Q}$ interval, $h(Q)$ is connected. If $Q$ is open, $Q$ can be written as an increasing sequence of compact intervals, and hence $h(Q)$ is connected. We may therefore assume that there is a point $p_{0} \in Q-Q^{0}$. Let $I=[a, b] \times[c, d]$ be an interval in $\bar{Q}$ which has $p_{0}$ as a vertex, say $p_{0}=(a, d)$. We only need show that $h\left(I^{0} \cup p_{0}\right)=h\left(I^{0}\right) \cup h\left(p_{0}\right)$ is connected.

Since $h(x, d)$ possesses the Darboux property, there is a sequence $\left\{x_{n}\right\}$ in $(a, b)$ such that $h\left(x_{n}, d\right) \rightarrow h(a, d)$. It suffices to show that for each $n$ there is a point $p_{n} \varepsilon I^{0}$ such that $\left|h\left(x_{n}, d\right)-h\left(p_{n}\right)\right|<1 / n$. There is $z_{n} \in(a, b)-x_{n}$ such that

$$
\left|h\left(x_{n}, d\right)-\frac{f\left(x_{n}, d\right)-f\left(z_{n}, d\right)}{x_{n}-z_{n}}\right|<\frac{1}{3 n} .
$$

By linear approximate continuity, there is $y_{n} \in(c, d)$ such that

$$
\left|f\left(x_{n}, d\right)-f\left(x_{n}, y_{n}\right)\right|<\frac{1}{3 n} \cdot\left|x_{n}-z_{n}\right|,\left|f\left(z_{n}, d\right)-f\left(z_{n}, y_{n}\right)\right|<\frac{1}{3 n} \cdot\left|x_{n}-z_{n}\right| \text {. }
$$

An easy calculation reveals that

$$
\left|h\left(x_{n}, d\right)-\frac{f\left(x_{n}, y_{n}\right)-f\left(z_{n}, y_{n}\right)}{x_{n}-z_{n}}\right|<\frac{1}{n} .
$$

By the MV-property for approximate derivatives, there is a point $\tau_{n}$ between $x_{n}, z_{n}$ such that

$$
f\left(x_{n}, y_{n}\right)-f\left(z_{n}, y_{n}\right)=h\left(\tau_{n}, y_{n}\right)\left(x_{n}-z_{n}\right) \text {. }
$$

Consequently, $\left|h\left(x_{n}, d\right)-h\left(\tau_{n}, y_{n}\right)\right|<1 / n$, and the proof is complete.

\section{BIBLIOGRAPHY}

1. A. Denjoy, Mémoire sur la totalization des nombres dérivés nonsommables, Ann. Sci. Ecole Norm. Sup. 33 (1916), 127-222.

2. A. Gleyzal, Interval functions, Duke Math. J. 8 (1941), 223-230.

3. C. Goffman and C. J. Neugebauer, On approximate derivatives, Proc. Amer. Math. Soc. 11 (1960), 962-966.

4. C. Goffman, C. J. Neugebauer and T. Nishiura, Density topology and approximate continuity, Duke Math. J. 28 (1961), 497-505.

5. C. Goffman and D. Waterman, Approximately continuous transformations, Proc. Amer. Math. Soc. 12 (1961), 116-121.

6. S. Saks, Theory of the integral, Warsaw, 1937.

7. A. Khintshine, Recherches sur la structure des fonctions mesurables, Fund. Math. 9 (1927), 217-279.

8. G. Tolstoff, Sur la dérivée approximative exacte, Mat. Sb. (N. S.) 4 (1938), 499-504.

9. L. Mišik, Der Mittelwertsatx für additive Intevallfunktionen, Fund. Math. 45 (1958), 64-70.

Purdue University,

LAFAYETTE, INDIANA 